\title{
Adaptive Synchronization between Fractional-Order Chaotic Real and Complex Systems with Unknown Parameters
}

\author{
Xiaomin Tian \\ School of Automation, Southeast University, Nanjing 210096, China \\ Correspondence should be addressed to Xiaomin Tian; tianxiaomin100@163.com \\ Received 26 May 2014; Revised 5 September 2014; Accepted 10 September 2014; Published 26 November 2014 \\ Academic Editor: Manuel De la Sen \\ Copyright (C) 2014 Xiaomin Tian. This is an open access article distributed under the Creative Commons Attribution License, which \\ permits unrestricted use, distribution, and reproduction in any medium, provided the original work is properly cited.

\begin{abstract}
The complex modified projective synchronization (CMPS) between fractional-order chaotic real and complex systems is investigated for the first time. The parameters of both master and slave systems are assumed to be unknown in advance; moreover, the slave system is perturbed by unknown but bounded external disturbances. The master and slave systems that achieved CMPS can be synchronized up to a complex constant matrix. On the basis of frequency distributed model of fractional integrator and Lyapunov stability theory, a robust adaptive control law is designed to realize the CMPS for two different types of fractional-order chaotic systems. Meanwhile, to deal with these unknown parameters, some fractional-order type parametric update laws are provided. An example is given to demonstrate the effectiveness and feasibility of the proposed synchronization scheme.
\end{abstract}

\section{Introduction}

Although fractional calculus is a mathematical topic with more than 300-year history, its applications in the fields of physics and engineering have attracted lots of attentions only in the recent years. It was found that, with the help of fractional calculus, many systems in interdisciplinary fields can be described more accurately. That is to say, fractional calculus provides a superb instrument for the description of memory and hereditary properties of various materials and processes. Many literatures have proven that some fractionalorder differential systems can behave chaotically [1-4].

The research on chaotic systems has grown significantly over the past decades and has become a popular topic. For example, Zhang and Yang [5] investigated a single driving variable approach to realize the adaptive stabilization of chaotic systems. Taghvafard and Erjaee [6] proposed an active control method to perform the phase and antiphase synchronization of two fractional-order chaotic systems. Aghababa [7] used finite-time theory to realize the finitetime synchronization of chaotic systems. Lu [8] developed a nonlinear observer to synchronize the chaotic systems. Chen et al. $[9,10]$ researched the chaos synchronization of fractional-order chaotic neural network. Recently, we [11] applied the sliding mode control strategy to stabilize a class of fractional-order chaotic systems with input nonlinearity.

However, all of the aforementioned researches only focus on the systems with real variables, while chaotic complex systems are not involved. Actually, chaotic complex systems can be widely used to describe a variety of physical phenomena, such as population inversion [12], detuned laser systems [13], and thermal convections of liquid flows [14]. At present, some control schemes have been proposed to synchronize two chaotic systems with complex variables [15-19]. But, it should be noted that all of chaotic complex nonlinear systems in the abovementioned literatures are integer-order systems. In recent years, several fractional-order chaotic complex systems are investigated [20-22]. Nevertheless, in [20-22], the systems' parameters are taken as known in the synchronization of two identical fractional-order chaotic complex systems. As a matter of fact, many systems' parameters cannot be exactly determined in advance. The synchronization will not be achieved under the effects of unknown uncertainties. Therefore, it is urgent to consider the impacts of unknown parameters in synchronizing two chaotic complex systems.

On the other hand, in many practical systems, the master (drive) system and slave (response) system may evolve in different directions with a constant intersection angle. Thus, 
the complex modified projective synchronization (CMPS) should be taken into consideration. This kind of CMPS is viewed as the generalization of several types of synchronization, such as complete synchronization (CS), antisynchronization (AS), modified projective synchronization (MPS), and projective synchronization (PS). However, to the best of our knowledge, up until now, there is no information available for the CMPS between fractional-order chaotic real and complex systems with unknown parameters.

Motivated by the above discussions, in this paper, the CMPS between two different fractional-order chaotic systems with unknown parameters and external disturbances is researched for the first time. Since the stability theory of integer-order system cannot be directly applied to fractionalorder systems, so, inspired by contributions from [23, 24], we use frequency distributed model of fractional integrator and Lyapunov stability theory to prove the stability of closed-loop system.

The rest of this paper is organized as follows. In Section 2, the relevant definitions and lemma are introduced. In Section 3, the adaptive controller and update laws of unknown parameters are designed in detail. Simulation results are presented in Section 4. Finally, conclusions are included in Section 5.

\section{Preliminaries}

The Grunwald-Letnikov definition, Riemann-Liouville definition, and Caputo definition are the most frequently used definitions for fractional calculus.

Definition 1. The $\alpha$ order Riemann-Liouville fractional integration is given by

$$
t_{0} I_{t}^{\alpha} f(t)=\frac{1}{\Gamma(\alpha)} \int_{t_{0}}^{t} \frac{f(\tau)}{(t-\tau)^{1-\alpha}} d \tau
$$

where $\Gamma(\cdot)$ is the Gamma function, determined by

$$
\Gamma(\alpha)=\int_{0}^{\infty} e^{-t} t^{\alpha-1} d t
$$

Definition 2. For $n-1<\alpha \leq n$, the Riemann-Liouville fractional derivative of order $\alpha$ is defined as

$$
\begin{aligned}
t_{0} D_{t}^{\alpha} f(t) & =\frac{d^{\alpha} f(t)}{d t^{\alpha}}=\frac{1}{\Gamma(n-\alpha)} \frac{d^{n}}{d t^{n}} \int_{t_{0}}^{t} \frac{f(\tau)}{(t-\tau)^{\alpha-n+1}} d \tau \\
& =\frac{d^{n}}{d t^{n}} I^{n-\alpha} f(t) .
\end{aligned}
$$

Definition 3. The Grunwald-Letnikov fractional derivative of order $\alpha$ is written as

$$
t_{0} D_{t}^{\alpha} f(t)=\lim _{h \rightarrow 0} \frac{1}{h^{\alpha}} \sum_{j=0}^{\left[\left(t-t_{0}\right) / h\right]}(-1)^{j}\left(\begin{array}{c}
\alpha \\
j
\end{array}\right) f(t-j h) .
$$

Definition 4. The Caputo fractional derivative of order $\alpha$ is defined as

$$
\begin{aligned}
& t_{0} D_{t}^{\alpha} f(t) \\
& \quad= \begin{cases}\frac{1}{\Gamma(m-\alpha)} \int_{t_{0}}^{t} \frac{f^{(m)}(\tau)}{(t-\tau)^{\alpha-m+1}} d \tau, & m-1<\alpha<m, \\
\frac{d^{m}}{d t^{m}} f(t), & \alpha=m,\end{cases}
\end{aligned}
$$

where $m$ is the smallest integer number, larger than $\alpha$.

In the rest of this paper, we will use the Caputo definition to describe the fractional chaotic systems and use the Grunwald-Letnikov approach to perform numerical simulations. For simplicity, in the rest of this paper, we use $D^{\alpha}$ instead of ${ }_{0} D_{t}^{\alpha}$.

Lemma 5 (see [25-27]). Consider a nonlinear fractionalorder system

$$
D^{\alpha} x(t)=f(x(t)),
$$

where $\alpha \in(0,1)$. Then the system is exactly equivalent to the following continuous frequency distributed model:

$$
\begin{gathered}
\frac{\partial z(\omega, t)}{\partial t}=-\omega z(\omega, t)+f(x(t)) \\
x(t)=\int_{0}^{\infty} \mu(\omega) z(\omega, t) d \omega,
\end{gathered}
$$

where $\mu(\omega)=((\sin (\alpha \pi)) / \pi) \omega^{-\alpha}$. In the above equations, $z(\omega, t)$ is the true state variable, and $x(t)$ is the pseudostate variable.

\section{Main Results}

Take the following $n$-dimensional chaotic real system as master system:

$$
D^{\alpha} x=F(x) \theta+f(x),
$$

where $x=\left(x_{1}, x_{2}, \ldots, x_{n}\right)^{T}$ is a real state vector. $F(x) \in R^{n \times m_{1}}$ is a real matrix and its elements are the functions of state variables. $\theta \in R^{m_{1}}$ is a vector of unknown parameters. $f(x) \epsilon$ $R^{n}$ is a vector of continuous nonlinear functions.

Consider the following $n$-dimensional chaotic complex system with external disturbances as slave system:

$$
D^{\alpha} y=G(y) \delta+g(y)+d(t)+W(t),
$$

where $y=\left(y_{1}, y_{2}, \ldots, y_{n}\right)^{T}$ is a complex state vector, and $y_{k}=y_{k}^{r}+j y_{k}^{i}, j=\sqrt{-1}$, and superscripts $r$ and $i$ are real and imaginary parts of state variables, respectively. $G(y) \in C^{n \times m_{2}}$ is a complex matrix and its elements are the functions of complex state variables. $\delta \in R^{m_{2}}$ (or $C^{m_{2}}$ ) is a vector of unknown parameters. $g(y) \in C^{n}$ is a vector of complex nonlinear functions. $d(t)=\left(d_{1}(t), d_{2}(t), \ldots, d_{n}(t)\right)^{T} \in C^{n}$ is a vector of external disturbances. $W(t)=\left(W_{1}(t), W_{2}(t), \ldots, W_{n}(t)\right)^{T}$ is a vector of controllers to be designed later. 
Remark 6. Most of fractional-order chaotic real and complex systems can be described by (8) and (9), such as fractionalorder real and complex Chen systems, fractional-order real and complex Lorenz systems, fractional-order Lu system, and fractional-order complex T system.

Before introducing our approach, we first give the definition of CMPS between systems (8) and (9).

Definition 7. Consider the real master system (8) and complex slave system (9), and the error of CMPS is defined as

$$
e=e^{r}+j e^{i}=y-H x=y^{r}-H^{r} x+j\left(y^{i}-H^{i} x\right),
$$

where $e^{r}=\left(e_{1}, e_{3}, \ldots, e_{2 n-1}\right)^{T}, e^{i}=\left(e_{2}, e_{4}, \ldots, e_{2 n}\right)^{T}, H=$ $\operatorname{diag}\left(h_{1}, h_{2}, \ldots, h_{n}\right)$, and $h_{k}=h_{k}^{r}+j h_{k}^{i}$. If $e \rightarrow 0$ as $t \rightarrow \infty$, then the CMPS between systems (8) and (9) is achieved.

Remark 8. Obviously, the problem of CMPS between (8) and (9) is equivalently transformed to the stabilization problem of error system.

Our goal in this paper is to design a robust adaptive controller to achieve the CMPS between fractional-order chaotic real and complex systems with unknown parameters.

In order to make the proposed method more reasonable and effective, an assumption is provided.

Assumption 9. In general, it is assumed that the external disturbances $d_{k}(t)=d_{k}^{r}(t)+j d_{k}^{i}(t)$ are bounded by

$$
\left|d_{k}(t)\right| \leq \phi_{k}
$$

where $k=1,2, \ldots, n .|\cdot|$ denotes the modulus of disturbance term and $\phi_{k}$ are known positive constants.

Theorem 10. Consider the real master system (8) and the complex slave system (9), if the controller is designed as

$$
\begin{gathered}
W_{k}(t)=W_{k}^{r}(t)+j W_{k}^{i}(t) \\
W_{k}^{r}(t)=-G_{k}^{r}(y) \widehat{\delta}+h_{k}^{r} F_{k}(x) \widehat{\theta}-g_{k}^{r}(y)+h_{k}^{r} f_{k}(x) \\
-L_{k} e_{2 k-1}-\xi_{k} \operatorname{sgn}\left(e_{2 k-1}\right) \\
W_{k}^{i}(t)=-G_{k}^{i}(y) \widehat{\delta}+h_{k}^{i} F_{k}(x) \hat{\theta}-g_{k}^{i}(y)+h_{k}^{i} f_{k}(x) \\
-L_{k} e_{2 k}-\xi_{k} \operatorname{sgn}\left(e_{2 k}\right),
\end{gathered}
$$

where $k=1,2, \ldots, n$. sgn is a sign function, $G_{k}^{r}(y), G_{k}^{i}(y)$, $g_{k}^{r}(y), g_{k}^{i}(y), F_{k}(x)$, and $f_{k}(x)$ are the kth row of $G^{r}(y), G^{i}(y)$, $g^{r}(y), g^{i}(y), F(x)$, and $f(x)$, respectively, $h_{k}^{r}$ and $h_{k}^{i}$ are given constants, and $L_{k}$ and $\xi_{k}$ are control gains, updated by

$$
\begin{gathered}
D^{\alpha} L_{k}=\beta\left(e_{2 k-1}^{2}+e_{2 k}^{2}\right) \\
D^{\alpha} \xi_{k}=\sigma\left(\left|e_{2 k-1}\right|+\left|e_{2 k}\right|\right)
\end{gathered}
$$

in which $\beta, \sigma$ are positive constants.

The adaptive rules of unknown parameters are selected as

$$
\begin{gathered}
D^{\alpha} \widehat{\theta}=-\left(H^{r} F(x)\right)^{T} e^{r}-\left(H^{i} F(x)\right)^{T} e^{i} \\
D^{\alpha} \widehat{\delta}=\left(G^{r}(y)\right)^{T} e^{r}+\left(G^{i}(y)\right)^{T} e^{i},
\end{gathered}
$$

where $\hat{\theta}$ and $\widehat{\delta}$ are the estimate values of $\theta$ and $\delta$, respectively. Then the CMPS between systems (8) and (9) can be achieved.

Proof. According to the definition of CMPS, we obtain

$$
\begin{aligned}
D^{\alpha} e= & D^{\alpha} e^{r}+j D^{\alpha} e^{i}=D^{\alpha} y^{r}-H^{r} D^{\alpha} x \\
& +j\left(D^{\alpha} y^{i}-H^{i} D^{\alpha} x\right) \\
= & G^{r}(y) \delta+g^{r}(y)+d^{r}(t)+W^{r}(t) \\
& -H^{r}(F(x) \theta+f(x)) \\
& +j\left[G^{i}(y) \delta+g^{i}(y)+d^{i}(t)+W^{i}(t)\right. \\
& \left.\quad-H^{i}(F(x) \theta+f(x))\right] .
\end{aligned}
$$

Namely, the real and imaginary parts of complex error system (15) and the adaptation laws (13) and (14) constitute the following closed-loop adaptive system:

$$
\begin{aligned}
& D^{\alpha} e_{2 k-1}= G_{k}^{r}(y) \delta+g_{k}^{r}(y)+d_{k}^{r}(t)+W_{k}^{r}(t) \\
&-h_{k}^{r}\left(F_{k}(x) \theta+f_{k}(x)\right) \\
& D^{\alpha} e_{2 k}= G_{k}^{i}(y) \delta+g_{k}^{i}(y)+d_{k}^{i}(t)+W_{k}^{i}(t) \\
&-h_{k}^{i}\left(F_{k}(x) \theta+f_{k}(x)\right) \\
& D^{\alpha} \widehat{\theta}=-\left(H^{r} F(x)\right)^{T} e^{r}-\left(H^{i} F(x)\right)^{T} e^{i} \\
& D^{\alpha} \widehat{\delta}=\left(G^{r}(y)\right)^{T} e^{r}+\left(G^{i}(y)\right)^{T} e^{i} \\
& D^{\alpha} L_{k}=\beta\left(e_{2 k-1}^{2}+e_{2 k}^{2}\right) \\
& D^{\alpha} \xi_{k}=\sigma\left(\left|e_{2 k-1}\right|+\left|e_{2 k}\right|\right) .
\end{aligned}
$$

According to the continuous frequency distributed model of fractional integrator [28-30], system (16) is exactly equivalent to the following infinite-dimensional ordinary differential equations:

$$
\begin{aligned}
& \frac{\partial z_{2 k-1}(\omega, t)}{\partial t}=-\omega z_{2 k-1}(\omega, t)+G_{k}^{r}(y) \delta+g_{k}^{r}(y)+d_{k}^{r}(t) \\
&+W_{k}^{r}(t)-h_{k}^{r}\left(F_{k}(x) \theta+f_{k}(x)\right) \\
& e_{2 k-1}=\int_{0}^{\infty} \mu(\omega) z_{2 k-1}(\omega, t) d \omega \\
& \frac{\partial z_{2 k}(\omega, t)}{\partial t}=-\omega z_{2 k}(\omega, t)+G_{k}^{i}(y) \delta+g_{k}^{i}(y)+d_{k}^{i}(t) \\
&+W_{k}^{i}(t)-h_{k}^{i}\left(F_{k}(x) \theta+f_{k}(x)\right) \\
& e_{2 k}=\int_{0}^{\infty} \mu(\omega) z_{2 k}(\omega, t) d \omega
\end{aligned}
$$

$$
\begin{gathered}
\frac{\partial z_{\widetilde{\theta}}(\omega, t)}{\partial t}=-\omega z_{\widetilde{\theta}}(\omega, t)-\left(H^{r} F(x)\right)^{T} e^{r}-\left(H^{i} F(x)\right)^{T} e^{i} \\
\widetilde{\theta}=\int_{0}^{\infty} \mu(\omega) z_{\widetilde{\theta}}(\omega, t) d \omega
\end{gathered}
$$




$$
\begin{gathered}
\frac{\partial z_{\widetilde{\delta}}(\omega, t)}{\partial t}=-\omega z_{\widetilde{\delta}}(\omega, t)+\left(G^{r}(y)\right)^{T} e^{r}+\left(G^{i}(y)\right)^{T} e^{i} \\
\tilde{\delta}=\int_{0}^{\infty} \mu(\omega) z_{\widetilde{\delta}}(\omega, t) d \omega \\
\frac{\partial z_{\widetilde{L}_{k}}(\omega, t)}{\partial t}=-\omega z_{\widetilde{L}_{k}}(\omega, t)+\beta\left(e_{2 k-1}^{2}+e_{2 k}^{2}\right) \\
\frac{\partial z_{\widetilde{\xi}_{k}}(\omega, t)}{\partial t}=-\omega z_{\widetilde{\xi}_{k}}(\omega, t)+\sigma\left(\left|e_{2 k-1}\right|+\left|e_{2 k}\right|\right) \\
\widetilde{\xi}_{k}=\int_{0}^{\infty} \mu(\omega) z_{\widetilde{L}_{k}}(\omega, t) d \omega
\end{gathered}
$$

where $k=1, \ldots, n \cdot \mu(\omega)=((\sin (\alpha \pi)) / \pi) \omega^{-\alpha}, \tilde{\theta}=\widehat{\theta}-\theta$, $\widetilde{\delta}=\widehat{\delta}-\delta, \widetilde{L}_{k}=L_{k}-L_{k}^{*}$, and $\widetilde{\xi}_{k}=\xi_{k}-\xi_{k}^{*}$ are estimation errors. $L_{k}^{*}$ and $\xi_{k}^{*}$ are positive constants to be determined later. In the above model, $z_{2 k-1}(\omega, t), z_{2 k}(\omega, t), z_{\widetilde{\theta}}(\omega, t), z_{\widetilde{\delta}}(\omega, t), z_{\widetilde{L}_{k}}(\omega, t)$, and $z_{\widetilde{\xi}_{k}}(\omega, t)$ are true state variables (vectors), while $e_{2 k-1}, e_{2 k}$, $\tilde{\theta}, \widetilde{\delta}, \widetilde{L}_{k}$, and $\widetilde{\xi}_{k}$ are pseudostate variables (vectors).

To prove the stability of (17), let us choose a positive definite Lyapunov function in the form of

$$
V(t)=V_{1}(t)+V_{2}(t)+V_{3}(t)+V_{4}(t)+V_{5}(t)+V_{6}(t),
$$

where

$$
\begin{gathered}
V_{1}(t)=\int_{0}^{\infty} \mu(\omega) v_{1}(\omega, t) d \omega, \\
v_{1}(\omega, t)=\frac{1}{2} \sum_{k=1}^{n} z_{2 k-1}^{2}(\omega, t), \\
V_{2}(t)=\int_{0}^{\infty} \mu(\omega) v_{2}(\omega, t) d \omega, \\
v_{2}(\omega, t)=\frac{1}{2} \sum_{k=1}^{n} z_{2 k}^{2}(\omega, t), \\
V_{3}(t)=\int_{0}^{\infty} \mu(\omega) v_{3}(\omega, t) d \omega, \\
v_{3}(\omega, t)=\frac{1}{2} z_{\widetilde{\theta}}^{T}(\omega, t) z_{\tilde{\theta}}(\omega, t), \\
V_{4}(t)=\int_{0}^{\infty} \mu(\omega) v_{4}(\omega, t) d \omega, \\
v_{4}(\omega, t)=\frac{1}{2} z_{\widetilde{\delta}}^{T}(\omega, t) z_{\widetilde{\delta}}(\omega, t), \\
v_{5}(t)=\int_{0}^{\infty} \mu(\omega) v_{5}(\omega, t) d \omega, \\
\frac{1}{2 \beta} \sum_{k=1}^{n} z_{\tilde{L}_{k}}^{2}(\omega, t),
\end{gathered}
$$

$$
\begin{aligned}
& V_{6}(t)=\int_{0}^{\infty} \mu(\omega) v_{6}(\omega, t) d \omega, \\
& v_{6}(\omega, t)=\frac{1}{2 \sigma} \sum_{k=1}^{n} z_{\widetilde{\xi}_{k}}^{2}(\omega, t) .
\end{aligned}
$$

Taking the derivative of $V(t)$ with respect to time, it yields

$$
\begin{aligned}
\frac{d V(t)}{d t}= & \int_{0}^{\infty} \mu(\omega) \sum_{k=1}^{n} z_{2 k-1} \frac{\partial z_{2 k-1}}{\partial t} d \omega \\
& +\int_{0}^{\infty} \mu(\omega) \sum_{k=1}^{n} z_{2 k} \frac{\partial z_{2 k}}{\partial t} d \omega+\int_{0}^{\infty} \mu(\omega) z_{\widetilde{\theta}}^{T} \frac{\partial z_{\widetilde{\theta}}}{\partial t} d \omega \\
& +\int_{0}^{\infty} \mu(\omega) z_{\widetilde{\delta}}^{T} \frac{\partial z_{\widetilde{\delta}}}{\partial t} d \omega \\
& +\frac{1}{\beta} \int_{0}^{\infty} \mu(\omega) \sum_{k=1}^{n} z_{\widetilde{L}_{k}} \frac{\partial z_{\widetilde{L}_{k}}}{\partial t} d \omega \\
& +\frac{1}{\sigma} \int_{0}^{\infty} \mu(\omega) \sum_{k=1}^{n} z_{\widetilde{\xi}_{k}} \frac{\partial z_{\widetilde{\xi}_{k}}}{\partial t} d \omega .
\end{aligned}
$$

Substituting (17) into (20), we have

$$
\begin{aligned}
& \frac{d V(t)}{d t}=\int_{0}^{\infty} \mu(\omega) \sum_{k=1}^{n} z_{2 k-1}\left[-\omega z_{2 k-1}+G_{k}^{r}(y) \delta\right. \\
& +g_{k}^{r}(y)+d_{k}^{r}(t)+W_{k}^{r}(t) \\
& \left.-h_{k}^{r}\left(F_{k}(x) \theta+f_{k}(x)\right)\right] d \omega \\
& +\int_{0}^{\infty} \mu(\omega) \sum_{k=1}^{n} z_{2 k}\left[-\omega z_{2 k}+G_{k}^{i}(y) \delta+g_{k}^{i}(y)\right. \\
& +d_{k}^{i}(t)+W_{k}^{i}(t) \\
& \left.-h_{k}^{i}\left(F_{k}(x) \theta+f_{k}(x)\right)\right] d \omega \\
& +\int_{0}^{\infty} \mu(\omega) z_{\widetilde{\theta}}^{T}\left[-\omega z_{\widetilde{\theta}}-\left(H^{r} F(x)\right)^{T} e^{r}\right. \\
& \left.-\left(H^{i} F(x)\right)^{T} e^{i}\right] d \omega \\
& +\int_{0}^{\infty} \mu(\omega) z_{\widetilde{\delta}}^{T}\left[-\omega z_{\widetilde{\delta}}+\left(G^{r}(y)\right)^{T} e^{r}\right. \\
& \left.+\left(G^{i}(y)\right)^{T} e^{i}\right] d \omega \\
& +\frac{1}{\beta} \int_{0}^{\infty} \mu(\omega) \sum_{i=1}^{n} z_{\widetilde{L}_{k}}\left[-\omega z_{\widetilde{L}_{k}}+\beta\left(e_{2 k-1}^{2}+e_{2 k}^{2}\right)\right] d \omega \\
& +\frac{1}{\sigma} \int_{0}^{\infty} \mu(\omega) \sum_{k=1}^{n} z_{\tilde{\xi}_{k}}\left[-\omega z_{\widetilde{\xi}_{k}}\right. \\
& \left.+\sigma\left(\left|e_{2 k-1}\right|+\left|e_{2 k}\right|\right)\right] d \omega \text {. }
\end{aligned}
$$


Inserting the control law (12) into (21), we obtain

$$
\begin{aligned}
& \frac{d V(t)}{d t}=-\int_{0}^{\infty} \mu(\omega) \omega \sum_{k=1}^{n} z_{2 k-1}^{2} d \omega \\
& +\sum_{k=1}^{n}\left[G_{k}^{r}(y)(\delta-\widehat{\delta})+d_{k}^{r}(t)-h_{k}^{r} F_{k}(x)(\theta-\widehat{\theta})\right. \\
& \left.-L_{k} e_{2 k-1}-\xi_{k} \operatorname{sgn}\left(e_{2 k-1}\right)\right] \\
& \times \int_{0}^{\infty} \mu(\omega) z_{2 k-1} d \omega-\int_{0}^{\infty} \mu(\omega) \omega \sum_{k=1}^{n} z_{2 k}^{2} d \omega \\
& +\sum_{k=1}^{n}\left[G_{k}^{i}(y)(\delta-\widehat{\delta})+d_{k}^{i}(t)-h_{k}^{i} F_{k}(x)(\theta-\widehat{\theta})\right. \\
& \left.-L_{k} e_{2 k}-\xi_{k} \operatorname{sgn}\left(e_{2 k}\right)\right] \\
& \times \int_{0}^{\infty} \mu(\omega) z_{2 k} d \omega-\int_{0}^{\infty} \mu(\omega) \omega z_{\widetilde{\theta}}^{T} z_{\tilde{\theta}} d \omega \\
& +\int_{0}^{\infty} \mu(\omega) z_{\tilde{\theta}}^{T} d \omega \\
& \times\left[-\left(H^{r} F(x)\right)^{T} e^{r}-\left(H^{i} F(x)\right)^{T} e^{i}\right] \\
& -\int_{0}^{\infty} \mu(\omega) \omega z_{\widetilde{\delta}}^{T} z_{\widetilde{\delta}} d \omega \\
& +\int_{0}^{\infty} \mu(\omega) z_{\widetilde{\delta}}^{T} d \omega\left[\left(G^{r}(y)\right)^{T} e^{r}+\left(G^{i}(y)\right)^{T} e^{i}\right] \\
& -\frac{1}{\beta} \int_{0}^{\infty} \mu(\omega) \omega \sum_{k=1}^{n} z_{\widetilde{L}_{k}}^{2} d \omega \\
& +\frac{1}{\beta} \sum_{k=1}^{n} \beta\left(e_{2 k-1}^{2}+e_{2 k}^{2}\right) \int_{0}^{\infty} \mu(\omega) z_{\widetilde{L}_{k}} d \omega \\
& -\frac{1}{\sigma} \int_{0}^{\infty} \mu(\omega) \omega \sum_{k=1}^{n} z_{\widetilde{\xi}_{k}}^{2} d \omega \\
& +\frac{1}{\sigma} \sum_{k=1}^{n} \sigma\left(\left|e_{2 k-1}\right|+\left|e_{2 k}\right|\right) \int_{0}^{\infty} \mu(\omega) z_{\widetilde{\xi}_{k}} d \omega .
\end{aligned}
$$

That is

$$
\begin{aligned}
\frac{d V(t)}{d t}=-J+\sum_{k=1}^{n}[ & G_{k}^{r}(y)(\delta-\widehat{\delta})+d_{k}^{r}(t) \\
& -h_{k}^{r} F_{k}(x)(\theta-\widehat{\theta}) \\
& \left.-L_{k} e_{2 k-1}-\xi_{k} \operatorname{sgn}\left(e_{2 k-1}\right)\right] e_{2 k-1}
\end{aligned}
$$

$$
\begin{aligned}
& +\sum_{k=1}^{n}\left[G_{k}^{i}(y)(\delta-\widehat{\delta})+d_{k}^{i}(t)-h_{k}^{i} F_{k}(x)(\theta-\widehat{\theta})\right. \\
& \left.\quad-L_{k} e_{2 k}-\xi_{k} \operatorname{sgn}\left(e_{2 k}\right)\right] e_{2 k} \\
& +\widetilde{\theta}^{T}\left[-\left(H^{r} F(x)\right)^{T} e^{r}-\left(H^{i} F(x)\right)^{T} e^{i}\right] \\
& +\widetilde{\delta}^{T}\left[\left(G^{r}(y)\right)^{T} e^{r}+\left(G^{i}(y)\right)^{T} e^{i}\right] \\
& +\sum_{k=1}^{n}\left(e_{2 k-1}^{2}+e_{2 k}^{2}\right) \widetilde{L}_{k}+\sum_{k=1}^{n}\left(\left|e_{2 k-1}\right|+\left|e_{2 k}\right|\right) \widetilde{\xi}_{k},
\end{aligned}
$$

where

$$
\begin{aligned}
J= & \sum_{k=1}^{n} \int_{0}^{\infty} \mu(\omega) \omega z_{2 k-1}^{2} d \omega+\sum_{k=1}^{n} \int_{0}^{\infty} \mu(\omega) \omega z_{2 k}^{2} d \omega \\
& +\int_{0}^{\infty} \mu(\omega) \omega z_{\widetilde{\theta}}^{T} z_{\widetilde{\theta}} d \omega+\int_{0}^{\infty} \mu(\omega) \omega z_{\widetilde{\delta}}^{T} z_{\widetilde{\delta}} d \omega \\
& +\sum_{k=1}^{n} \frac{1}{\beta} \int_{0}^{\infty} \mu(\omega) \omega z_{\widetilde{L}_{k}}^{2} d \omega+\sum_{k=1}^{n} \frac{1}{\sigma} \int_{0}^{\infty} \mu(\omega) \omega z_{\widetilde{\xi}_{k}}^{2} d \omega>0
\end{aligned}
$$

Therefore,

$$
\begin{aligned}
\sum_{k=1}^{n} & {\left[G_{k}^{r}(y)(\delta-\widehat{\delta})\right] e_{2 k-1}+\sum_{k=1}^{n}\left[G_{k}^{i}(y)(\delta-\widehat{\delta})\right] e_{2 k} } \\
& =\left(e^{r}\right)^{T}\left[G^{r}(y)(\delta-\widehat{\delta})\right]+\left(e^{i}\right)^{T}\left[G^{i}(y)(\delta-\widehat{\delta})\right] \\
& =(\delta-\widehat{\delta})^{T}\left[\left(G^{r}(y)\right)^{T} e^{r}+\left(G^{i}(y)\right)^{T} e^{i}\right] \\
& =-\widetilde{\delta}^{T}\left[\left(G^{r}(y)\right)^{T} e^{r}+\left(G^{i}(y)\right)^{T} e^{i}\right]
\end{aligned}
$$

$\sum_{k=1}^{n}\left[-h_{k}^{r} F_{k}(x)(\theta-\hat{\theta})\right] e_{2 k-1}+\sum_{k=1}^{n}\left[-h_{k}^{i} F_{k}(x)(\theta-\hat{\theta})\right] e_{2 k}$

$$
\begin{aligned}
& =\left(e^{r}\right)^{T}\left[-H^{r} F(x)(\theta-\hat{\theta})\right]+\left(e^{i}\right)^{T}\left[-H^{i} F(x)(\theta-\hat{\theta})\right] \\
& =(\theta-\widehat{\theta})^{T}\left[-\left(H^{r} F(x)\right)^{T} e^{r}-\left(H^{i} F(x)\right)^{T} e^{i}\right] \\
& =-\widetilde{\theta}^{T}\left[-\left(H^{r} F(x)\right)^{T} e^{r}-\left(H^{i} F(x)\right)^{T} e^{i}\right] .
\end{aligned}
$$


On the basis of (25), then (23) can be rewritten as

$$
\begin{aligned}
\frac{d V(t)}{d t}= & -J+\sum_{k=1}^{n} d_{k}^{r}(t) e_{2 k-1}+\sum_{k=1}^{n} d_{k}^{i}(t) e_{2 k} \\
& -\sum_{k=1}^{n} L_{k}^{*}\left(e_{2 k-1}^{2}+e_{2 k}^{2}\right)-\sum_{k=1}^{n} \xi_{k}^{*}\left(\left|e_{2 k-1}\right|+\left|e_{2 k}\right|\right) \\
\leq & -J+\sum_{k=1}^{n}\left|d_{k}^{r}(t)\right|\left|e_{2 k-1}\right| \\
& +\sum_{k=1}^{n}\left|d_{k}^{i}(t)\right|\left|e_{2 k}\right|-L^{*} E^{T} E-\xi^{*}\|E\| \\
\leq & -J+\sum_{k=1}^{n} \phi_{k}\left|e_{2 k-1}\right| \\
& +\sum_{k=1}^{n} \phi_{k}\left|e_{2 k}\right|-L^{*} E^{T} E-\xi^{*}\|E\| \\
\leq & -J-\left(\xi^{*}-\phi\right)\|E\|-L^{*} E^{T} E,
\end{aligned}
$$

where $E=\left(e_{1}, e_{3}, \ldots, e_{2 n-1}, e_{2}, e_{4}, \ldots, e_{2 n}\right)^{T}, \xi^{*}=\min \left\{\xi_{k}^{*}\right\}$, $\phi=\max \left\{\phi_{k}\right\}, L^{*}=\min \left\{L_{k}^{*}\right\}$, and $k=1,2, \ldots, n$. It is obvious that when $\xi^{*} \geq \phi, L^{*}>0$, we have

$$
\frac{d V(t)}{d t} \leq-J-L^{*} E^{T} E<0 .
$$

According to the conclusions of [23], the fractional-order closed-loop adaptive system (16) is asymptotically stable. This proves that the CMPS between systems (8) and (9) can be achieved by using the control law (12) and the fractional adaptation laws (13) and (14). Therefore, the proof is completed.

Remark 11. Apparently, the theoretical results in $[15,31]$ are the special cases of our scheme. It should be noted that the feedback control gain in practical applications is desired as small as possible; however, the theoretical feedback control gains in $[15,31]$ are fixed values no matter where the initial values start; thus, the gains must be larger than the values needed, which means a kind of waste in practice. In our method, we use an adaptive controller to overcome the above drawbacks. The control gains $L_{k}, \xi_{k}$ can be automatically adapted to the suitable values, which can simplify the design process and reduce the cost of control.

If $\delta$ is a complex unknown vector and can be written as $\delta=\delta^{r}+j \delta^{i}$, then slave system can be rewritten as

$$
\begin{aligned}
D^{\alpha} y= & G(y)\left(\delta^{r}+j \delta^{i}\right)+g(y)+d(t)+W(t) \\
= & G^{r}(y) \delta^{r}-G^{i}(y) \delta^{i}+g^{r}(y)+d^{r}(t)+W^{r}(t) \\
& +j\left(G^{r}(y) \delta^{i}+G^{i}(y) \delta^{r}+g^{i}(y)+d^{i}(t)+W^{i}(t)\right) .
\end{aligned}
$$

According to Definition 7, we can obtain

$$
\begin{aligned}
D^{\alpha} e= & G^{r}(y) \delta^{r}-G^{i}(y) \delta^{i}+g^{r}(y)+d^{r}(t) \\
& +W^{r}(t)-H^{r}(F(x) \theta+f(x)) \\
& +j\left[G^{r}(y) \delta^{i}+G^{i}(y) \delta^{r}+g^{i}(y)+d^{i}(t)\right. \\
& \left.+W^{i}(t)-H^{i}(F(x) \theta+f(x))\right] .
\end{aligned}
$$

That is, the real and imaginary parts of (29) can be represented as

$$
\begin{aligned}
D^{\alpha} e_{2 k-1}= & G_{k}^{r}(y) \delta^{r}-G_{k}^{i}(y) \delta^{i}+g_{k}^{r}(y)+d_{k}^{r}(t) \\
& +W_{k}^{r}(t)-h_{k}^{r}\left(F_{k}(x) \theta+f_{k}(x)\right) \\
D^{\alpha} e_{2 k}= & G_{k}^{r}(y) \delta^{i}+G_{k}^{i}(y) \delta^{r}+g_{k}^{i}(y)+d_{k}^{i}(t) \\
& +W_{k}^{i}(t)-h_{k}^{i}\left(F_{k}(x) \theta+f_{k}(x)\right) .
\end{aligned}
$$

Similarly, for stabilizing the error system (30), we give the following theorem.

Theorem 12. Consider the real master system (8) and the complex slave system (28), if the controller is designed as

$$
\begin{aligned}
W_{k}(t)= & W_{k}^{r}(t)+W_{k}^{i}(t) \\
W_{k}^{r}(t)= & -G_{k}^{r}(y) \widehat{\delta}^{r}+G_{k}^{i}(y) \widehat{\delta}^{i}+h_{k}^{r} F_{k}(x) \hat{\theta}-g_{k}^{r}(y) \\
& +h_{k}^{r} f_{k}(x)-L_{k} e_{2 k-1}-\xi_{k} \operatorname{sgn}\left(e_{2 k-1}\right) \\
W_{k}^{i}(t)= & -G_{k}^{r}(y) \hat{\delta}^{i}-G_{k}^{i}(y) \hat{\delta}^{r}+h_{k}^{i} F_{k}(x) \hat{\theta}-g_{k}^{i}(y) \\
& +h_{k}^{i} f_{k}(x)-L_{k} e_{2 k}-\xi_{k} \operatorname{sgn}\left(e_{2 k}\right),
\end{aligned}
$$

where $k=1,2, \ldots, n . L_{k}$ and $\xi_{k}$ are control gains, which are updated by

$$
\begin{aligned}
D^{\alpha} L_{k} & =\beta\left(e_{2 k-1}^{2}+e_{2 k}^{2}\right) \\
D^{\alpha} \xi_{k} & =\sigma\left(\left|e_{2 k-1}\right|+\left|e_{2 k}\right|\right),
\end{aligned}
$$

where $\beta$ and $\sigma$ are positive constants.

The parametric update laws are designed as

$$
\begin{aligned}
D^{\alpha} \widehat{\theta} & =-\left(H^{r} F(x)\right)^{T} e^{r}-\left(H^{i} F(x)\right)^{T} e^{i} \\
D^{\alpha} \widehat{\delta}^{r} & =\left(G^{r}(y)\right)^{T} e^{r}+\left(G^{i}(y)\right)^{T} e^{i} \\
D^{\alpha} \widehat{\delta}^{i} & =\left(-G^{i}(y)\right)^{T} e^{r}+\left(G^{r}(y)\right)^{T} e^{i} .
\end{aligned}
$$

Then the CMPS between systems (8) and (28) can be achieved.

Proof. It is similar to that of Theorem 10. Limited by the length of this paper, the proof is omitted here.

In this paper, for simplicity, we merely consider the case of real unknown parameters. 


\section{Simulation Example}

In this example, we take fractional-order real Chen system as master system, described by

$$
\begin{aligned}
& D^{\alpha} x_{1}=a_{1}\left(x_{2}-x_{1}\right) \\
& D^{\alpha} x_{2}=\left(a_{2}-a_{1}\right) x_{1}+a_{2} x_{2}-x_{1} x_{3} \\
& D^{\alpha} x_{3}=x_{1} x_{2}-a_{3} x_{3},
\end{aligned}
$$

where $x=\left(x_{1}, x_{2}, x_{3}\right)^{T}$ is a real state vector, and

$$
\begin{aligned}
& F(x)=\left(\begin{array}{ccc}
x_{2}-x_{1} & 0 & 0 \\
-x_{1} & x_{1}+x_{2} & 0 \\
0 & 0 & -x_{3}
\end{array}\right), \\
& f(x)=\left(\begin{array}{c}
0 \\
-x_{1} x_{3} \\
x_{1} x_{2}
\end{array}\right) \quad \theta=\left(\begin{array}{l}
a_{1} \\
a_{2} \\
a_{3}
\end{array}\right) .
\end{aligned}
$$

Take the fractional-order complex Lorenz system with external disturbances as slave system, written as

$$
\begin{aligned}
& D^{\alpha} y_{1}=b_{1}\left(y_{2}-y_{1}\right)+d_{1}(t)+W_{1}(t) \\
& D^{\alpha} y_{2}=b_{2} y_{1}-y_{2}-y_{1} y_{3}+d_{2}(t)+W_{2}(t) \\
& D^{\alpha} y_{3}=\frac{1}{2}\left(\bar{y}_{1} y_{2}+y_{1} \bar{y}_{2}\right)-b_{3} y_{3}+d_{3}(t)+W_{3}(t),
\end{aligned}
$$

where $y_{1}=u_{1}+j u_{2}$ and $y_{2}=u_{3}+j u_{4}$ are two complex state variables, $y_{3}=u_{5}$ is a real state variable, and

$$
\begin{aligned}
& G(y)=\left(\begin{array}{ccc}
y_{2}-y_{1} & 0 & 0 \\
0 & y_{1} & 0 \\
0 & 0 & -y_{3}
\end{array}\right)=\left(\begin{array}{ccc}
u_{3}-u_{1} & 0 & 0 \\
0 & u_{1} & 0 \\
0 & 0 & -u_{5}
\end{array}\right) \\
&+j\left(\begin{array}{ccc}
u_{4}-u_{2} & 0 & 0 \\
0 & u_{2} & 0 \\
0 & 0 & 0
\end{array}\right) \\
& g(y)=\left(\begin{array}{c}
-y_{2}-y_{1} y_{3} \\
\frac{1}{2}\left(\bar{y}_{1} y_{2}+y_{1} \bar{y}_{2}\right)
\end{array}\right)=\left(\begin{array}{c}
0 \\
-u_{3}-u_{1} u_{5} \\
u_{1} u_{3}+u_{2} u_{4}
\end{array}\right) \\
&+j\left(\begin{array}{c}
-u_{4}-u_{2} u_{5} \\
0
\end{array}\right) \\
& d(t)=\left(\begin{array}{c}
0.2 \sin (\pi t) \\
0.2 \sin (0.5 \pi t) \\
0.2 \sin (0.5 \pi t)
\end{array}\right)+j\left(\begin{array}{c}
0.2 \cos (\pi t) \\
0.2 \cos (\pi t) \\
0
\end{array}\right), \\
& \delta=\left(\begin{array}{l}
b_{1} \\
b_{2} \\
b_{3}
\end{array}\right) .
\end{aligned}
$$

In this simulation, the complex scaling matrix $H=$ $\operatorname{diag}\left(h_{1}, h_{2}, h_{3}\right)$ and $h_{1}=h_{1}^{r}+j h_{1}^{i}, h_{2}=h_{2}^{r}+j h_{2}^{i}$ are two complex constants, and $h_{3}$ is a real constant. According to Theorem 10, the controller can be designed as

$$
\begin{aligned}
& W_{1}^{r}(t)=-\widehat{b}_{1}\left(u_{3}-u_{1}\right)+\widehat{a}_{1} h_{1}^{r}\left(x_{2}-x_{1}\right)-L_{1} e_{1}-\xi_{1} \operatorname{sgn}\left(e_{1}\right) \\
& \begin{aligned}
W_{1}^{i}(t)= & -\widehat{b}_{1}\left(u_{4}-u_{2}\right)+\widehat{a}_{1} h_{1}^{i}\left(x_{2}-x_{1}\right)-L_{1} e_{2}-\xi_{1} \operatorname{sgn}\left(e_{2}\right) \\
W_{2}^{r}(t)= & -\widehat{b}_{2} u_{1}+h_{2}^{r}\left(-\widehat{a}_{1} x_{1}+\widehat{a}_{2}\left(x_{1}+x_{2}\right)\right)-\left(-u_{3}-u_{1} u_{5}\right) \\
& +h_{2}^{r}\left(-x_{1} x_{3}\right)-L_{2} e_{3}-\xi_{2} \operatorname{sgn}\left(e_{3}\right) \\
W_{2}^{i}(t)= & -\widehat{b}_{2} u_{2}+h_{2}^{i}\left(-\widehat{a}_{1} x_{1}+\widehat{a}_{2}\left(x_{1}+x_{2}\right)\right)-\left(-u_{4}-u_{2} u_{5}\right) \\
& +h_{2}^{i}\left(-x_{1} x_{3}\right)-L_{2} e_{4}-\xi_{2} \operatorname{sgn}\left(e_{4}\right) \\
W_{3}(t)= & \widehat{b}_{3} u_{5}-h_{3} \widehat{a}_{3} x_{3}-\left(u_{1} u_{3}+u_{2} u_{4}\right)+h_{3} x_{1} x_{2} \\
& -L_{3} e_{5}-\xi_{3} \operatorname{sgn}\left(e_{5}\right) .
\end{aligned}
\end{aligned}
$$
of

The adaptation laws (13) and (14) are obtained in the form

$$
\begin{gathered}
D^{\alpha} L_{1}=\beta\left(e_{1}^{2}+e_{2}^{2}\right), \quad D^{\alpha} L_{2}=\beta\left(e_{3}^{2}+e_{4}^{2}\right), \\
D^{\alpha} L_{3}=\beta e_{5}^{2} \\
D^{\alpha} \xi_{1}=\sigma\left(\left|e_{1}\right|+\left|e_{2}\right|\right), \quad D^{\alpha} \xi_{2}=\sigma\left(\left|e_{3}\right|+\left|e_{4}\right|\right), \\
D^{\alpha} \xi_{3}=\sigma\left|e_{5}\right| \\
D^{\alpha} \widehat{a}_{1}=-h_{1}^{r}\left(x_{2}-x_{1}\right) e_{1}+h_{2}^{r} x_{1} e_{3}-h_{1}^{i}\left(x_{2}-x_{1}\right) e_{2}+h_{2}^{i} x_{1} e_{4} \\
D^{\alpha} \widehat{a}_{2}=-h_{2}^{r}\left(x_{1}+x_{2}\right) e_{3}-h_{2}^{i}\left(x_{1}+x_{2}\right) e_{4} \\
D^{\alpha} \widehat{a}_{3}=h_{3} x_{3} e_{5} \\
D^{\alpha} \widehat{b}_{1}=\left(u_{3}-u_{1}\right) e_{1}+\left(u_{4}-u_{2}\right) e_{2} \\
D^{\alpha} \widehat{b}_{2}=u_{1} e_{3}+u_{2} e_{4} \\
D^{\alpha} \widehat{b}_{3}=-u_{5} e_{5} .
\end{gathered}
$$

Letting $\alpha=0.998, H=\operatorname{diag}(1+j, 1+j, 1)$, the unknown parameters are $\theta=(35,28,3)^{T}$ and $\delta=(10,28,8 / 3)^{T}$, and the initial values can be randomly chosen as $x(t)=x\left(0^{+}\right)=$ $(2,2,2)^{T}, y(t)=y\left(0^{+}\right)=(1+j, 1+j, 1)^{T}, \widehat{\theta}(t)=\widehat{\theta}\left(0^{+}\right)=$ $(0,0,0)^{T}, \widehat{\delta}(t)=\widehat{\delta}\left(0^{+}\right)=(0,0,0)^{T}, L(t)=L\left(0^{+}\right)=(0,0,0)^{T}$, and $\xi(t)=\xi\left(0^{+}\right)=(0,0,0)^{T}$ (where $L(t)=\left(L_{1}, L_{2}, L_{3}\right)^{T}$ and $\left.\xi(t)=\left(\xi_{1}, \xi_{2}, \xi_{3}\right)^{T}\right)$ for $-\infty \leq t \leq 0$. The positive constants are as follows: $\beta=10$ and $\sigma=0.1$. The chaotic attractors of systems (34) and (36) without control are displayed in Figures 1 and 2 . 


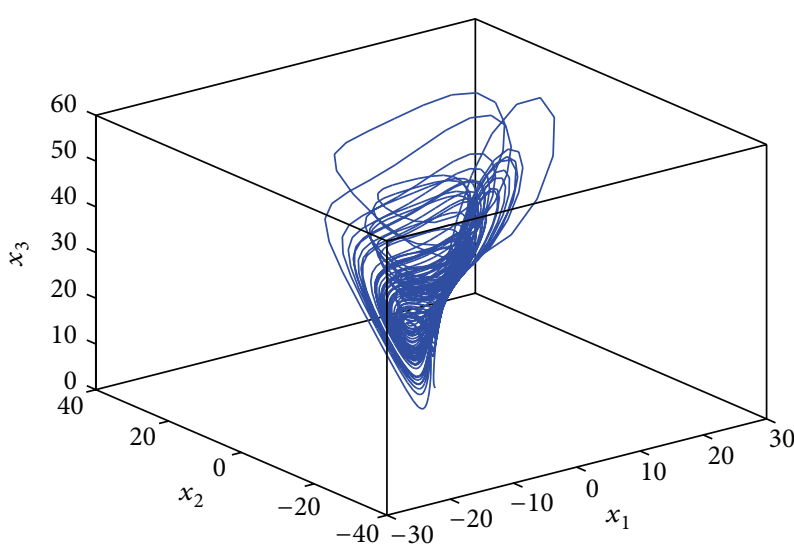

Figure 1: The 3D projection of chaotic attractor of (34).
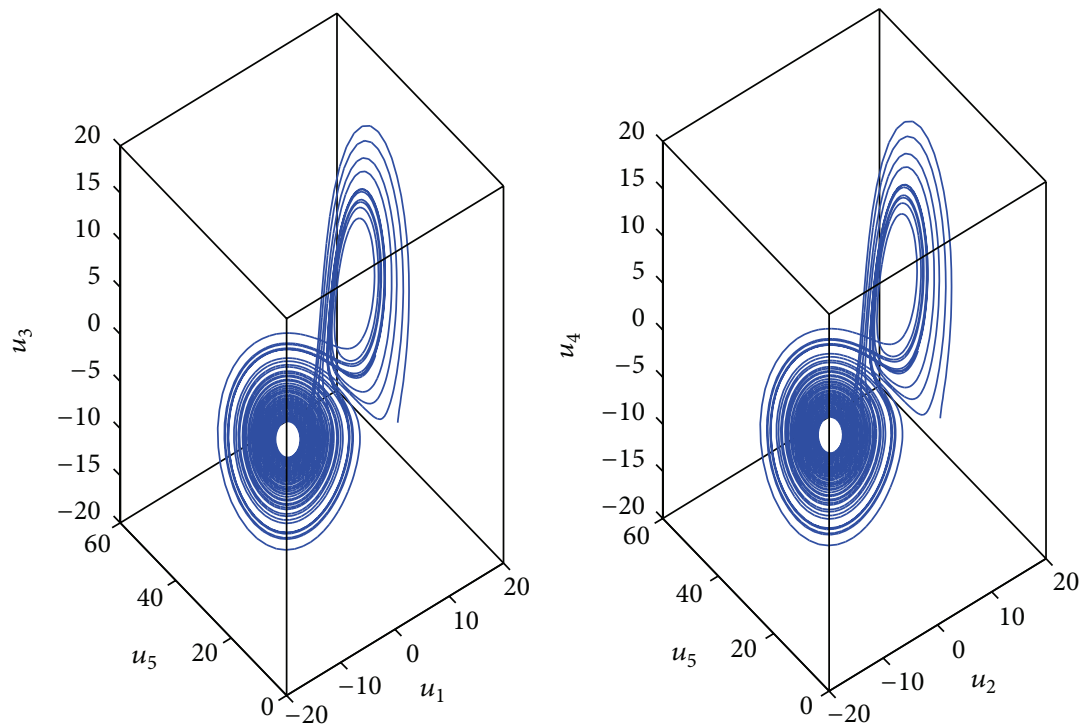

FIgURE 2: The 3D projections of chaotic attractors of (36) without control.

When the controller is activated, the time evolutions of CMPS errors between systems (34) and (36) are shown in Figure 3. It is clear that all error states asymptotically converge to zero.

The time responses of estimated parameter vectors $\widehat{\theta}$ and $\widehat{\delta}$ in master and slave systems are illustrated in Figure 4 . It is obvious that all unknown parameters gradually converge to their actual values.

All simulation results imply that the proposed control scheme is effective in synchronizing fractional-order chaotic real system and fractional-order chaotic complex system in the sense of CMPS.

Remark 13. In above simulation example, the random choice of complex matrix $H$ will not affect the theoretical results.

\section{Conclusions}

In this paper, the problem of complex modified projective synchronization (CMPS) between fractional-order real and complex systems is investigated. The parameters of both master and slave systems are assumed to be unknown in advance. Since the effects of external disturbances are fully taken into consideration, the proposed approach is more practical and meaningful than that of the existing methods. In order to prove the stability of the closed-loop system, the frequency distributed model of fractional integrator and Lyapunov stability theory are used. The simulation results have verified the effectiveness and applicability of the proposed synchronization scheme. Because our results contain and extend most existing works, we believe that there are high potentials in the proposed method. 

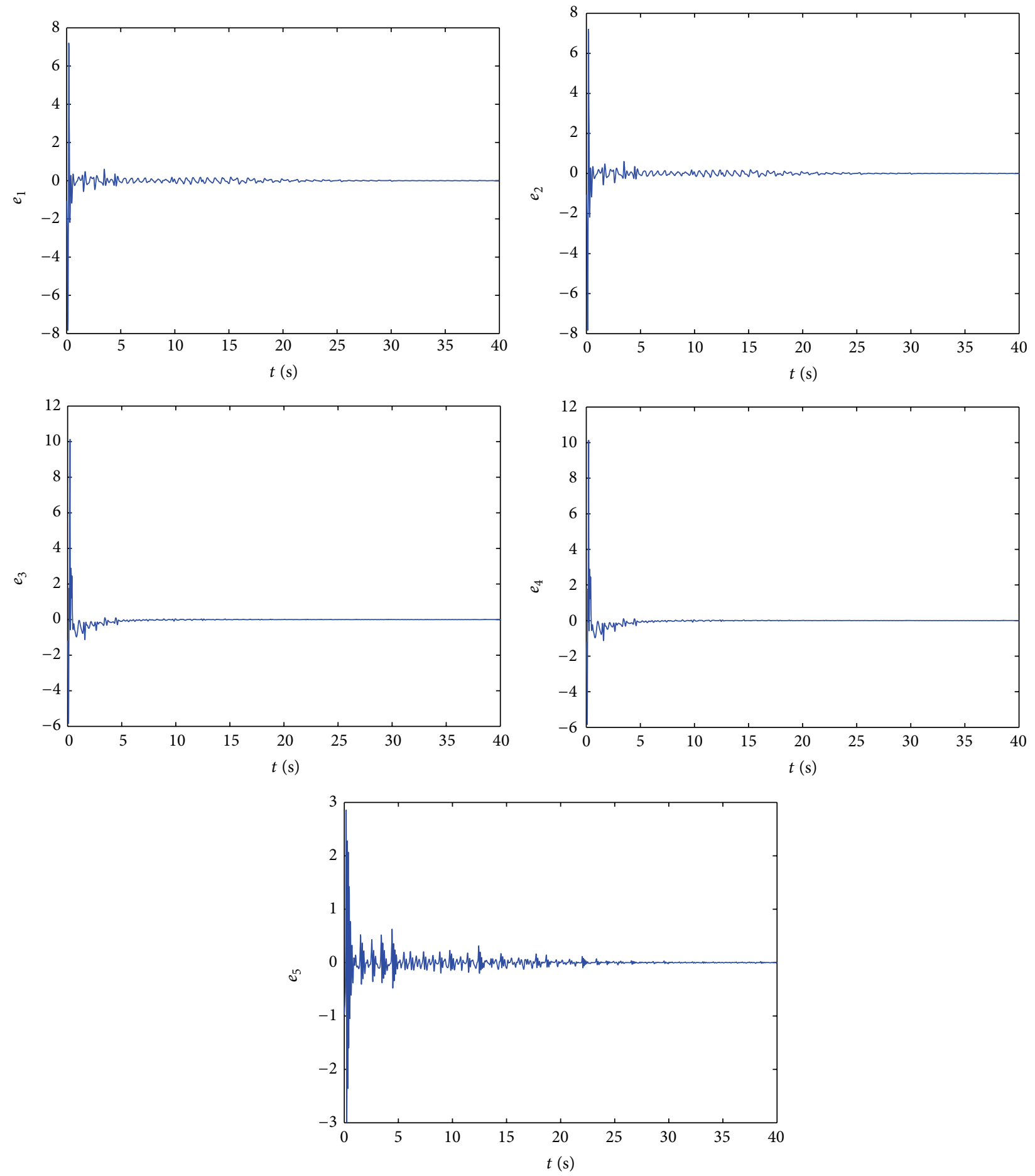

FIgURE 3: Time evolutions of CMPS errors between systems (34) and (36).

\section{Conflict of Interests}

The author declares that there is no conflict of interests regarding the publication of this paper.

\section{Acknowledgments}

The author would like to thank the reviewers and editors for many suggestions that helped improve the paper. The author 

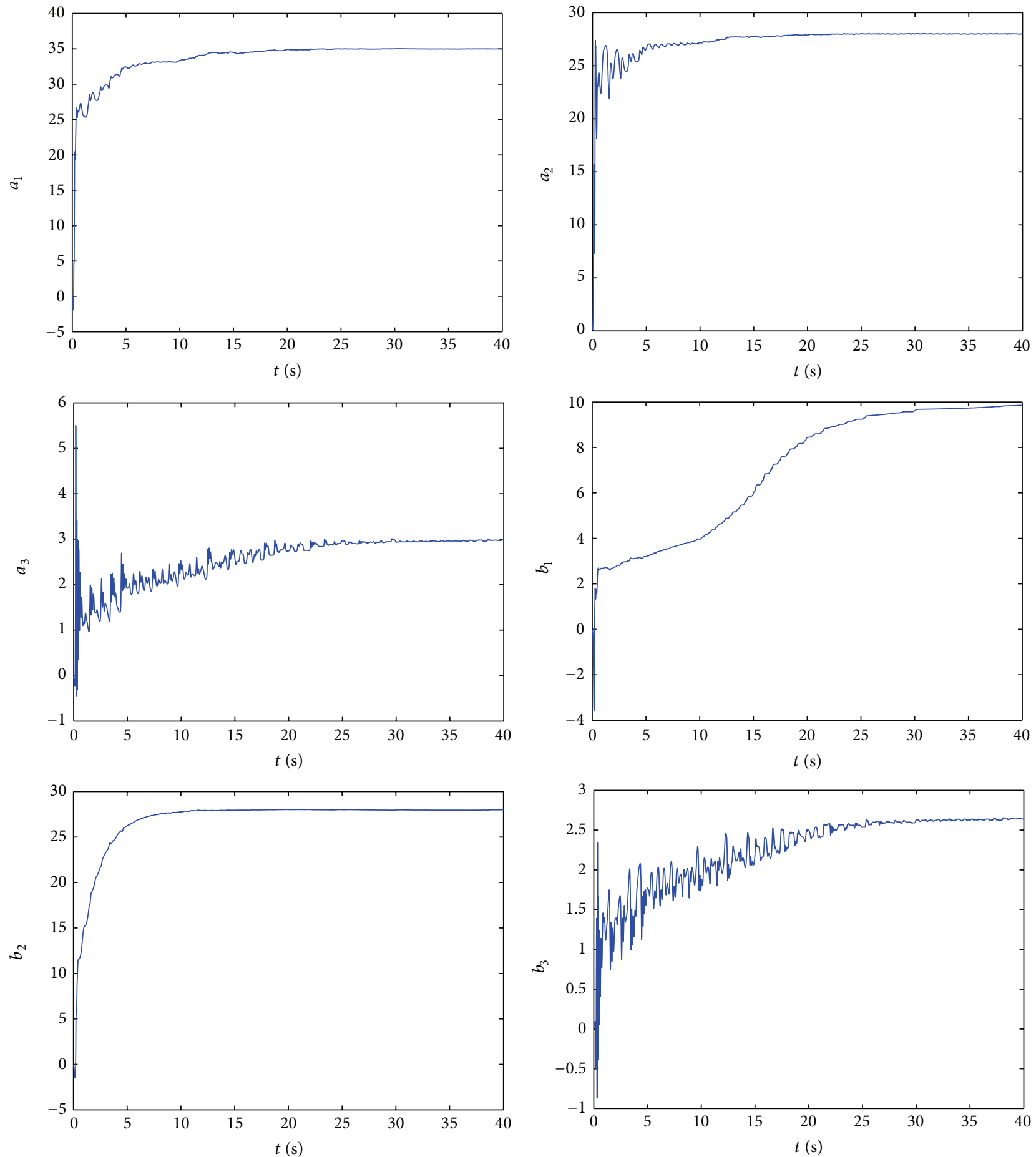

FIgURE 4: Time responses of estimate parameters in systems (34) and (36).

also wants to thank Professor Shumin Fei for his help in the revision of this paper. This research was supported by the National Natural Science Foundation of China (61273119 and 61374038) and the Natural Science Foundation of Jiangsu Province of China (BK2011253).

\section{References}

[1] C. M. Chang and H. K. Chen, "Chaos and hybrid projective synchronization of commensurate and incommensurate fractional-order Chen-Lee systems," Nonlinear Dynamics, vol. 62, no. 4, pp. 851-858, 2010. 
[2] I. Grigorenko and E. Grigorenko, "Chaotic dynamics of the fractional Lorenz system,” Physical Review Letters, vol. 91, no. 3, Article ID 034101, 2003.

[3] X. Wu and Y. Lu, "Generalized projective synchronization of the fractional-order Chen hyperchaotic system," Nonlinear Dynamics, vol. 57, no. 1-2, pp. 25-35, 2009.

[4] R. Zhang and S. Yang, "Robust chaos synchronization of fractional-order chaotic systems with unknown parameters and uncertain perturbations," Nonlinear Dynamics, vol. 69, no. 3, pp. 983-992, 2012.

[5] R. X. Zhang and S. P. Yang, "Stabilization of fractional-order chaotic system via a single state adaptive-feedback controller," Nonlinear Dynamics, vol. 68, no. 1-2, pp. 45-51, 2012.

[6] H. Taghvafard and G. H. Erjaee, "Phase and anti-phase synchronization of fractional order chaotic systems via active control," Communications in Nonlinear Science and Numerical Simulation, vol. 16, no. 10, pp. 4079-4088, 2011.

[7] M. P. Aghababa, "Finite-time chaos control and synchronization of fractional-order nonautonomous chaotic (hyperchaotic) systems using fractional nonsingular terminal sliding mode technique," Nonlinear Dynamics, vol. 69, no. 1-2, pp. 247-267, 2012.

[8] J. G. Lu, "Nonlinear observer design to synchronize fractionalorder chaotic systems via a scalar transmitted signal," Physica A: Statistical Mechanics and Its Applications, vol. 359, no. 1-4, pp. 107-118, 2006.

[9] L. P. Chen, J. F. Qu, Y. Chai, R. C. Wu, and G. Y. Qi, "Synchronization of a class of fractional-order chaotic neural networks," Entropy, vol. 15, no. 8, pp. 3265-3276, 2013.

[10] L. Chen, Y. Chai, R. Wu, J. Sun, and T. Ma, "Cluster synchronization in fractional-order complex dynamical networks," Physics Letters A, vol. 376, no. 35, pp. 2381-2388, 2012.

[11] X. Tian and S. Fei, "Robust control of a class of uncertain fractional-order chaotic systems with input nonlinearity via an adaptive sliding mode technique," Entropy, vol. 16, no. 2, pp. 729-746, 2014.

[12] G. M. Mahmoud, M. A. Al-Kashif, and A. A. Farghaly, "Chaotic and hyperchaotic attractors of a complex nonlinear system," Journal of Physics A: Mathematical and Theoretical, vol. 41, no. 5, Article ID 055104, 2008.

[13] E. Roldán, G. J. de Valcárcel, R. Vilaseca, and P. Mandel, "Singlemode-laser phase dynamics," Physical Review A, vol. 48, no. 1, pp. 591-598, 1993.

[14] V. Y. Toronov and V. L. Derbov, "Boundedness of attractors in the complex Lorenz model," Physical Review E. Statistical, Nonlinear, and Soft Matter Physics, vol. 55, no. 3, pp. 3689-3692, 1997.

[15] G. M. Mahmoud and E. E. Mahmoud, "Complex modified projective synchronization of two chaotic complex nonlinear systems," Nonlinear Dynamics, vol. 73, no. 4, pp. 2231-2240, 2013.

[16] G. M. Mahmoud and E. E. Mahmoud, "Phase and antiphase synchronization of two identical hyperchaotic complex nonlinear systems," Nonlinear Dynamics, vol. 61, no. 1-2, pp. 141-152, 2010.

[17] Q. Wei, X. Y. Wang, and X. P. Hu, "Feedback chaotic synchronization of a complex chaotic system with disturbances," Journal of Vibration and Control, 2014.

[18] X. Y. Wang and N. Wei, "Modified function projective lag synchronization of hyperchaotic complex systems with parameter perturbations and external perturbations," Journal of Vibration and Control, 2014.
[19] X. B. Zhou, M. R. Jiang, and X. M. Cai, "Synchronization of a novel hyperchaotic complex-variable system based on finitetime stability theory," Entropy, vol. 15, no. 10, pp. 4334-4344, 2013.

[20] C. Luo and X. Y. Wang, "Chaos in the fractional-order complex Lorenz system and its synchronization," Nonlinear Dynamics, vol. 71, no. 1-2, pp. 241-257, 2013.

[21] C. Luo and X. Wang, "Chaos generated from the fractionalorder complex Chen system and its application to digital secure communication," International Journal of Modern Physics C, vol. 24, no. 4, Article ID 1350025, 23 pages, 2013.

[22] X. Liu, L. Hong, and L. Yang, "Fractional-order complex T system: bifurcations, chaos control, and synchronization," Nonlinear Dynamics, vol. 75, no. 3, pp. 589-602, 2014.

[23] J. Yuan, B. Shi, and W. Q. Ji, "Adaptive sliding mode control of a novel class of fractional chaotic systems," Advances in Mathematical Physics, vol. 2013, Article ID 576709, 13 pages, 2013.

[24] B. Shi, J. Yuan, and C. Dong, "On fractional model reference adaptive control," The Scientific World Journal, vol. 2014, Article ID 521625, 8 pages, 2014.

[25] J. C. Trigeassou, N. Maamri, J. Sabatier, and A. Oustaloup, "A Lyapunov approach to the stability of fractional differential equations," Signal Processing, vol. 91, no. 3, pp. 437-445, 2011.

[26] J. Sabatier, M. Merveillaut, R. Malti, and A. Oustaloup, "On a representation of fractional order systems: interests for the initial condition problem," in Proceedings of the 3rd IFAC Workshop on Fractional Differentiation and its Applications (FDA '08), Ankara, Turkey, November 2008.

[27] J. Sabatier, M. Merveillaut, R. Malti, and A. Oustaloup, "How to impose physically coherent initial conditions to a fractional system?" Communications in Nonlinear Science and Numerical Simulation, vol. 15, no. 5, pp. 1318-1326, 2010.

[28] J. C. Trigeassou, N. Maamri, J. Sabatier, and A. Oustaloup, "State variables and transients of fractional order differential systems," Computers \& Mathematics with Applications, vol. 64, no. 10, pp. 3117-3140, 2012.

[29] J. C. Trigeassou, N. Maamri, J. Sabatier, and A. Oustaloup, "Transients of fractional-order integrator and derivatives," Signal, Image and Video Processing, vol. 6, no. 3, pp. 359-372, 2012.

[30] J. C. Trigeassou and N. Maamri, "Initial conditions and initialization of linear fractional differential equations," Signal Processing, vol. 91, no. 3, pp. 427-436, 2011.

[31] K. M. Abualnaja and E. E. Mahmoud, "Analytical and numerical study of the projective synchronization of the chaotic complex nonlinear systems with uncertain parameters and its applications in secure communication," Mathematical Problems in Engineering, vol. 2014, Article ID 808375, 10 pages, 2014. 


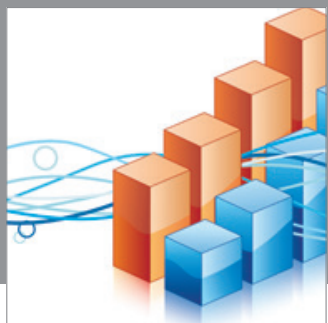

Advances in

Operations Research

mansans

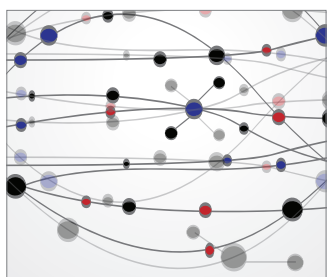

The Scientific World Journal
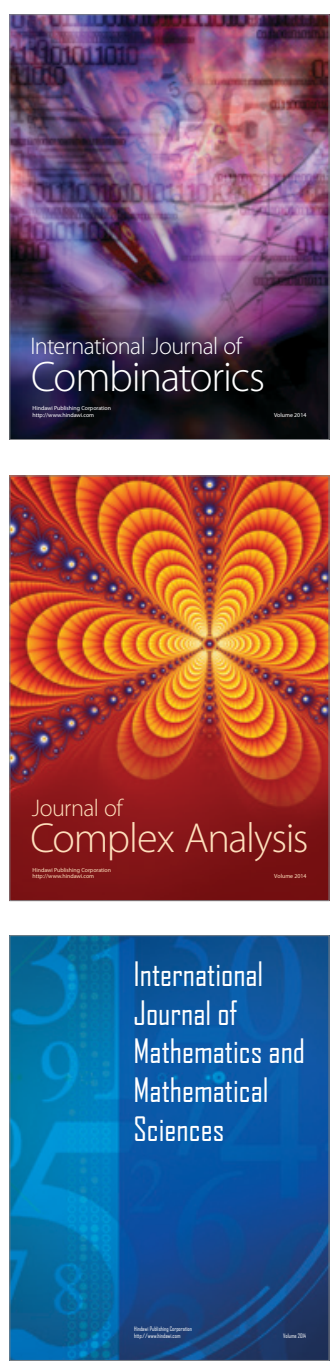
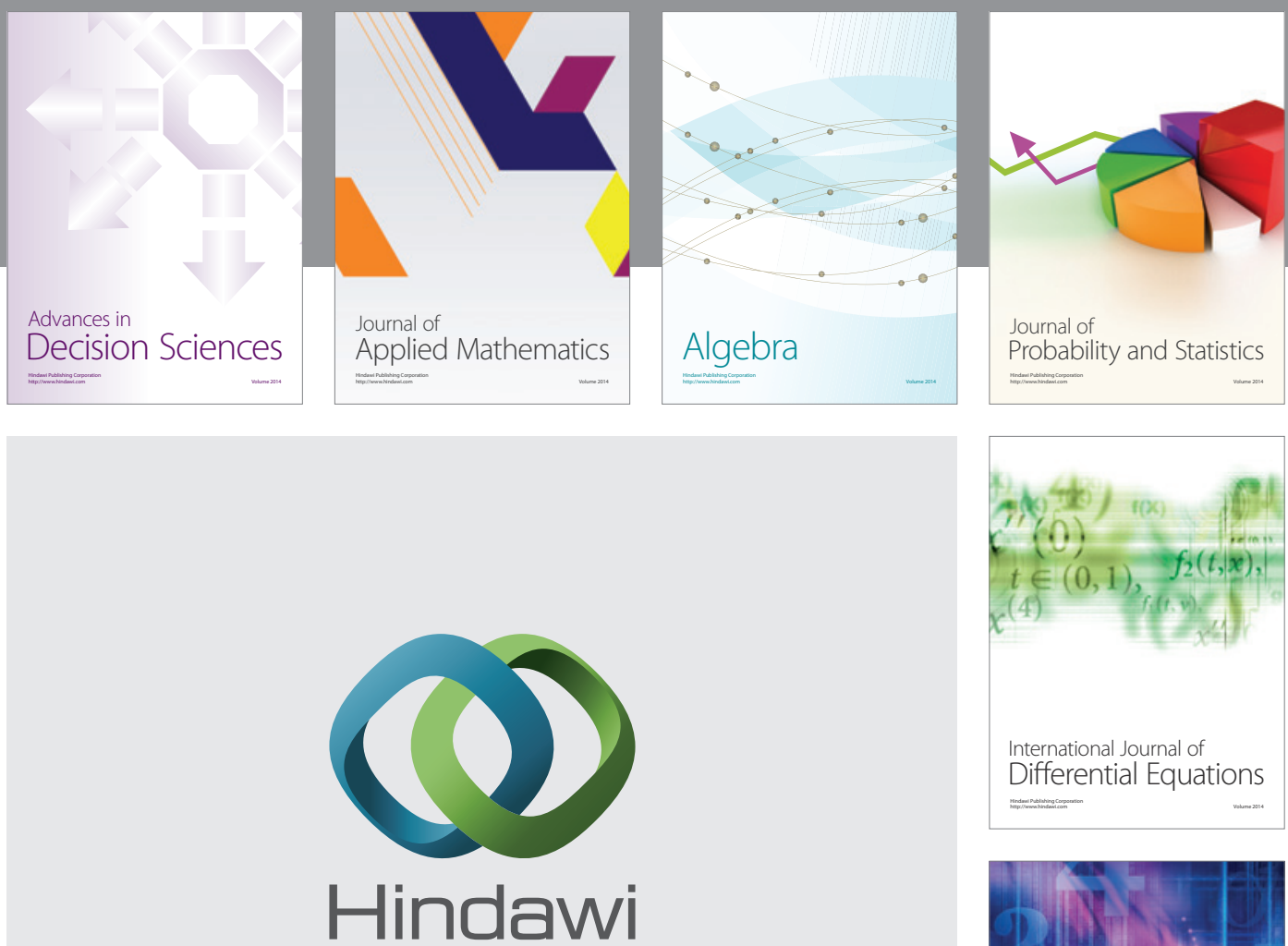

Submit your manuscripts at http://www.hindawi.com
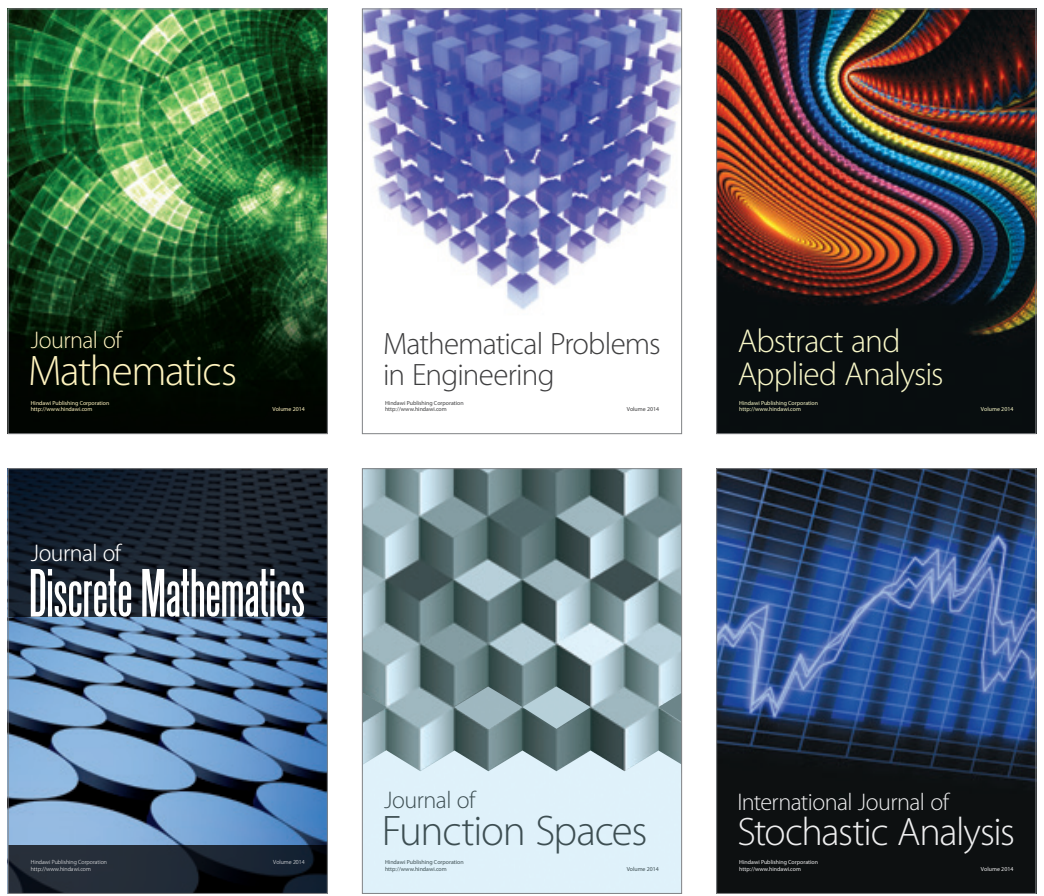

Journal of

Function Spaces

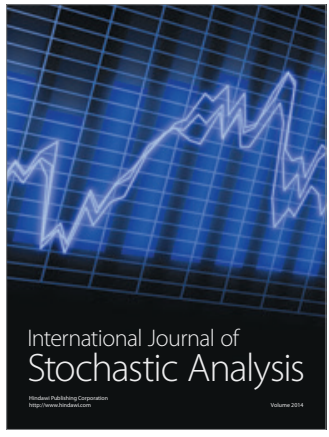

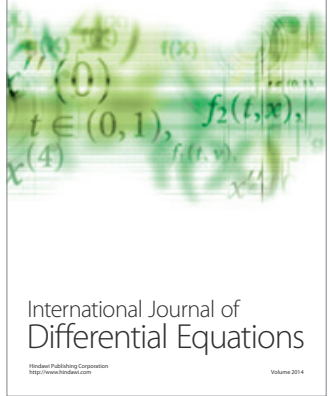
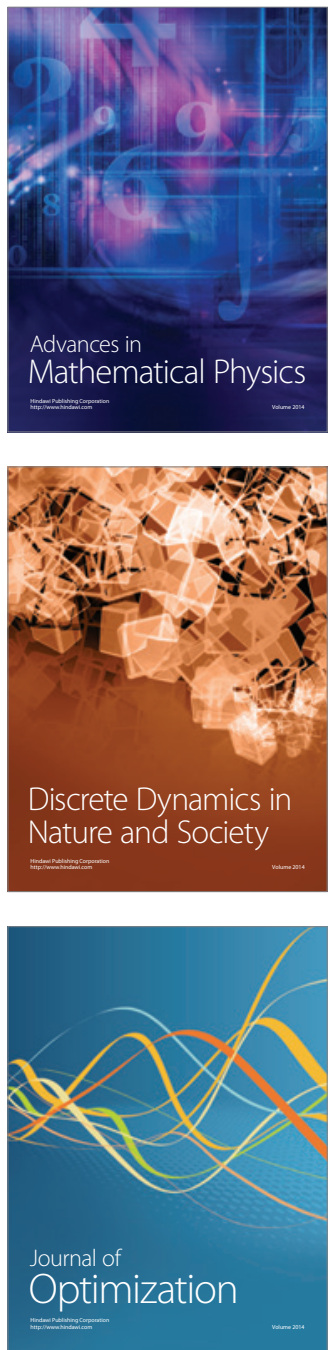